\title{
Mauke beim Pferd
}

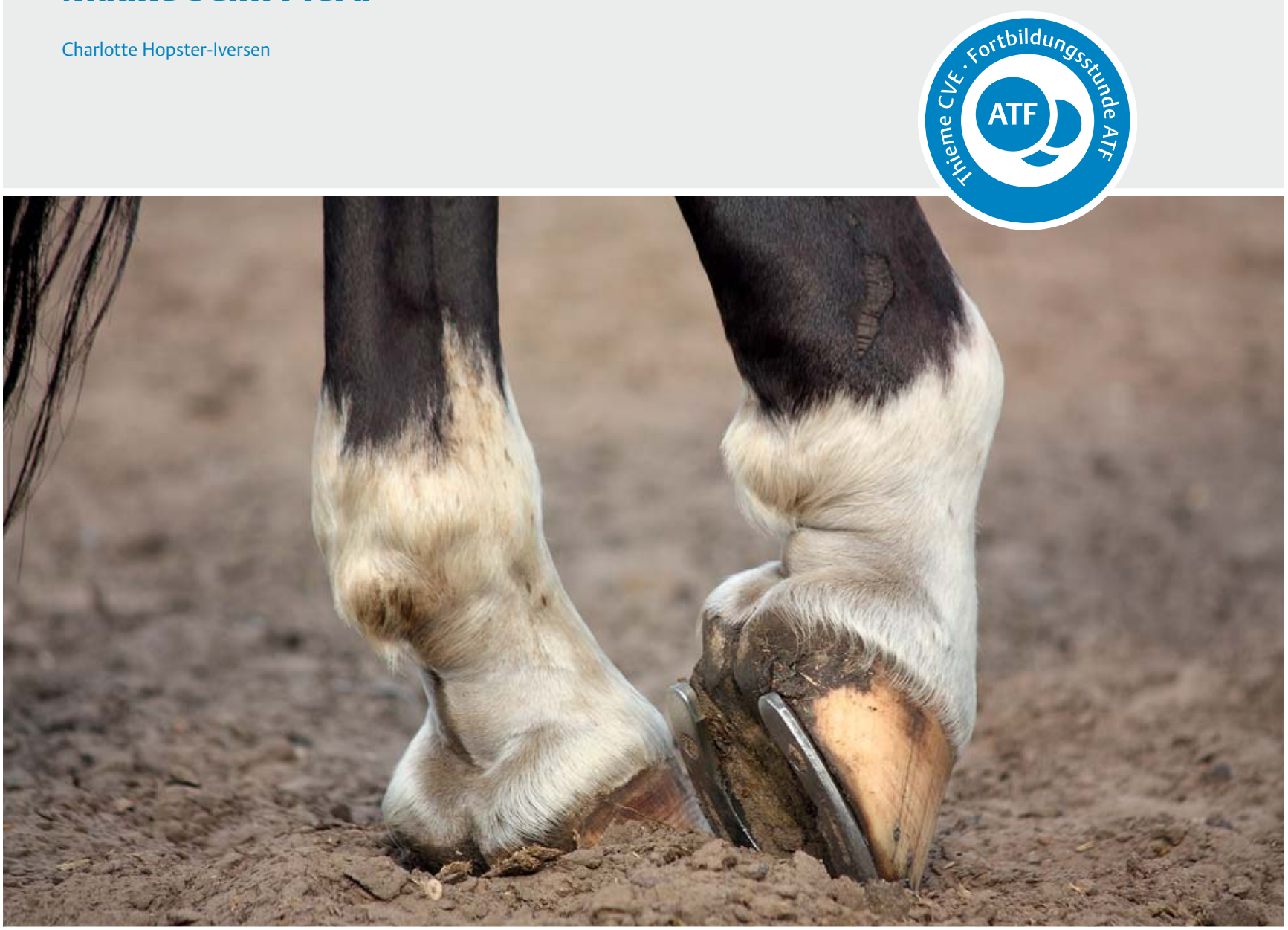

(c) virgonira - stock.adobe.com

Das klinische Bild einer Mauke ist vielgestaltig und erschwert somit die diagnostische Abklärung. Wurde eine Diagnose gestellt, ist die Therapie dennoch häufig langwierig und die Rezidivgefahr hoch. Ausschlaggebend für den Therapieerfolg sind eine Kombination aus Haltungsoptimierung zusammen mit einer gezielten und konsequenten lokalen Behandlung.

\section{Definition}

Eine Dermatitis in der Fesselbeuge wird als Mauke bezeichnet. Dabei ist sie mehr als Syndrom und nicht als Diagnose zu verstehen, um eine klinische Symptomatik mit vielen möglichen Ätiologien bei ähnlichem Erscheinungsbild zu beschreiben. Im englischen Sprachgebrauch wird Mauke als equine pastern dermatitis und ferner auch als mud fever oder grease heel bezeichnet.

\section{Prävalenz}

Mauke tritt häufig beim Pferd auf und kommt weltweit vor. Bei Kaltblütern sowie bei anderen Pferden mit Behang tritt sie gehäuft auf [1,2]. In einer Studie an 3-jährigen Pferden der Rasse Freiberger betrug die Prävalenz $15,2 \%$ [3], während bei deutschen Kaltblütern über 2,5 Jahre die Prävalenz zwischen 47-84\% lag [4]. Dabei stieg die Prävalenz mit zunehmendem Alter. Als weiterer prädisponierender Faktor wurden in der Studie von Federici et al. [3] weiße Gliedmaßen identifiziert (hier besteht eine 2,6-fach höhere Prävalenz für Mauke). 
- Tab. 1 Prädisponierende, primäre und aufrechterhaltende Faktoren der Mauke.

\begin{tabular}{|c|c|c|}
\hline prädisponierende Faktoren & primäre Faktoren & aufrechterhaltende Faktoren \\
\hline $\begin{array}{l}\text { genetisch } \\
\text { - unpigmentierte Haut und Haare der distalen Gliedmaße } \\
\text { - chronisch progressives Lymphödem bei manchen Kaltblut- } \\
\text { rassen } \\
\text { - Fesselbehang } \\
\text { - Verhornungsstörung }\end{array}$ & $\begin{array}{l}\text { physikalische oder chemische Irritatio- } \\
\text { nen } \\
\text { " behandelte Einstreu } \\
\text { " Blistern } \\
\text { - Motoröl }\end{array}$ & $\begin{array}{l}\text { Sekundärinfektionen } \\
\text { - Staphylococcus spp. } \\
\text { - Dermatophilus congolensis } \\
\text { - fusiforme Bakterien } \\
\text { - Malassezia spp. (Botryomykose) }\end{array}$ \\
\hline $\begin{array}{l}\text { Umgebung } \\
\text { - Klima oder Feuchtigkeit } \\
\text { - mangelhafte Stall- oder Weidehygiene } \\
\text { - alkalischer Boden } \\
\text { - Sand }\end{array}$ & $\begin{array}{l}\text { immunmediiert } \\
\text { - Allergie (Insekt/Kontakt) } \\
\text { - Vaskulitis } \\
\text { - Pemphigus }\end{array}$ & $\begin{array}{l}\text { pathologische Hautveränderungen } \\
\text { - Trauma } \\
\text { - Insektenbisse } \\
\text { - Culicoides spp. } \\
\text { - Zeckenbisse }\end{array}$ \\
\hline \multirow[t]{3}{*}{$\begin{array}{l}\text { iatrogen } \\
\text { - irritierende topische Produkte (z. B. Teebaumöl) } \\
\text { - Trainingsvorrichtungen } \\
\text { - ineffizientes Putzen des Pferdes }\end{array}$} & $\begin{array}{l}\text { infektiös } \\
\text { - Dermatophyten (Spirochäten) }\end{array}$ & $\begin{array}{l}\text { Umgebung } \\
\text { - UV-Licht } \\
\text { - Kälte }\end{array}$ \\
\hline & $\begin{array}{l}\text { parasitär } \\
\text { - Chorioptes spp. } \\
\text { - Trombiculosis (Herbstgrasmilbe) } \\
\text { - (Pelodera strongyloides) } \\
\text { - (Strongyloides-westeri-Larven) }\end{array}$ & \\
\hline & $\begin{array}{l}\text { neoplastisch } \\
\text { - Sarkoide }\end{array}$ & \\
\hline
\end{tabular}

\section{Ätiologie und Pathogenese}

Die Mauke ist eine multifaktorielle Erkrankung. Dabei kommt es durch das Zusammenspiel von prädisponierenden, primären und aufrechterhaltenden Faktoren ( $\triangleright$ Tab. 1) zur Entstehung einer Dermatitis, die in ihrer Ausprägung oft sehr unterschiedlich sein kein ( $\triangleright$ Abb. 1 und 2). Erschwerend kommt hinzu, dass tierärztlicher Rat zumeist erst im Verlauf der Erkrankung eingeholt wird, nachdem diverse Therapien vom Besitzer selbst ausprobiert wurden. Dadurch ist das klinische Bild oft sehr variabel und meistens durch sekundäre Veränderungen beeinflusst.

Ätiologisch kommen neben Umweltfaktoren diverse Auslöser infrage, wie z. B. bakterielle Infektionen (Staphylococcus spp., Dermatophilose), Dermatophytose, Milbenbefall, Kontaktdermatitis oder Vaskulitis (idiopathisch oder bedingt durch Photosensibilisierung).

Als Sonderform des Maukekomplexes tritt die pastern leukocytoclastic vasculitis auf. Sie ist eine vermutlich immun-mediierte, photodynamisch aktivierte, kutane Vaskulitis an unpigmentierten Hautarealen der distalen Gliedmaßen. Sie tritt vor allem im Sommer auf und ist durch gut umschriebene, zirkuläre, schmerzhafte, erythomatöse, exsudative Krusten gekennzeichnet [2] ( Abb. 3).

Beim Kaltblut ist darüber hinaus eine Sonderform bekannt: die chronische Warzenmauke (verrucous pododer-

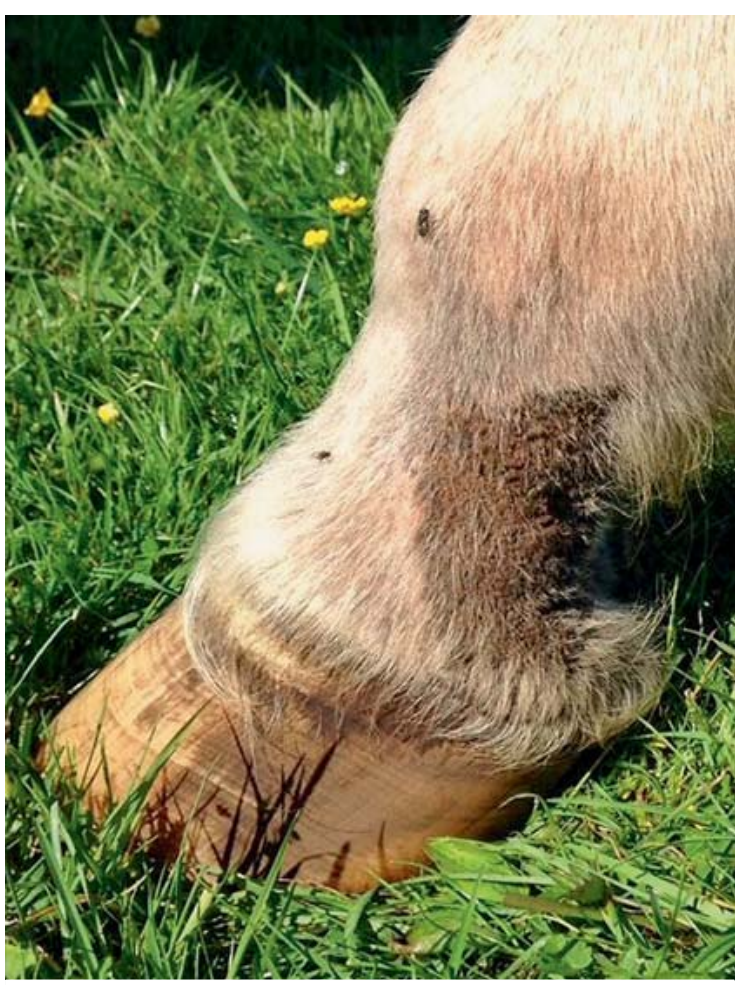

- Abb. 1 Krustöse Veränderungen im Bereich der Fesselbeuge bei einem Warmblutpferd. @ Charlotte HopsterIversen 


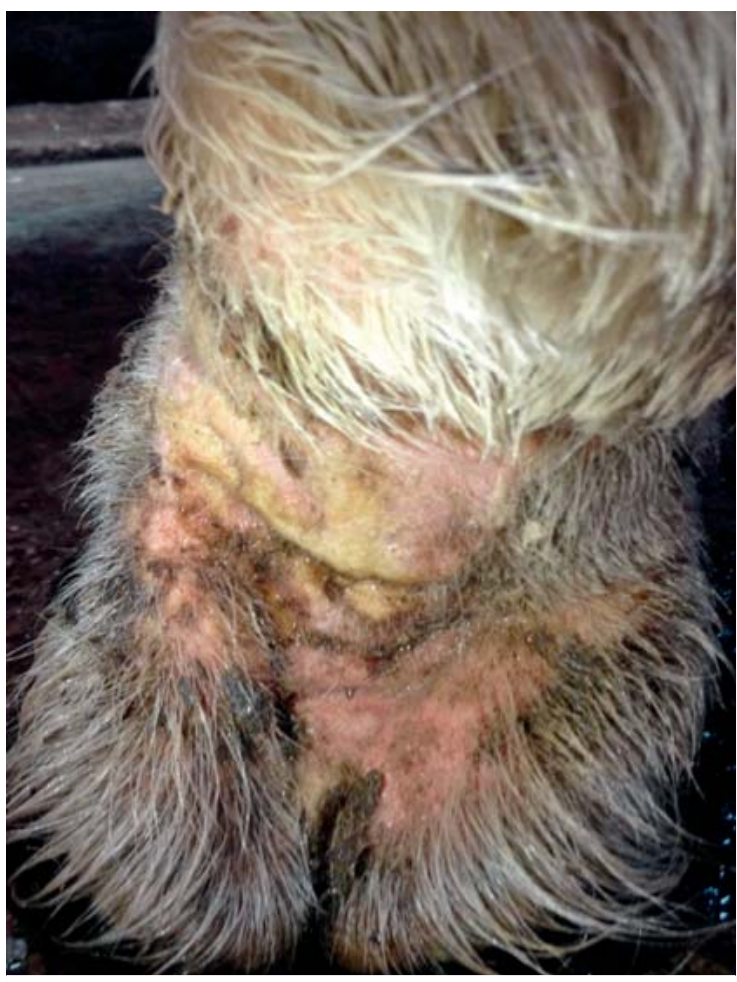

- Abb. 2 Exsudative Veränderungen in der Fesselbeuge bei einem Warmblutpferd im Bereich der weißen Abzeichen der Gliedmaße (Pferd aus $>$ Abb. 1). @ C Charlotte Hopster-Iversen matitis). Bei der Warzenmauke führen unzureichende Lymphabflusssysteme der distalen Gliedmaße zu hochgradigen Schwellungen, zu Fibrosierungen, einer komprimierten Immunabwehr sowie sekundären Hautinfektionen $[2,5,12]$.

\section{Merke}

Das klinische Bild der Mauke ist vielgestaltig: prädisponierende, primäre sowie aufrechterhaltende Faktoren spielen in Pathogenese und Symptomatik eine bedeutende Rolle.

\section{Klinik}

Die Hautläsionen bei Mauke treten zumeist bilateral symmetrisch in der Fesselbeuge auf ( $\bullet$ Abb. 1), es kann jedoch auch nur eine Gliedmaße betroffen sein. Häufiger sind die Hintergliedmaßen sowie weiße Gliedmaßen betroffen ( $\triangleright$ Abb. 2). Die Ausbreitung ist variabel mit teilweiser Einbeziehung der gesamten distalen Gliedmaße vom Kronrand bis zum Tarsus bzw. Karpus.

Die Warzenmauke ( $\triangleright$ Abb. 4) kann auch im Bereich der Karpal- und Tarsalbeuge auftreten, verbunden mit Quer-

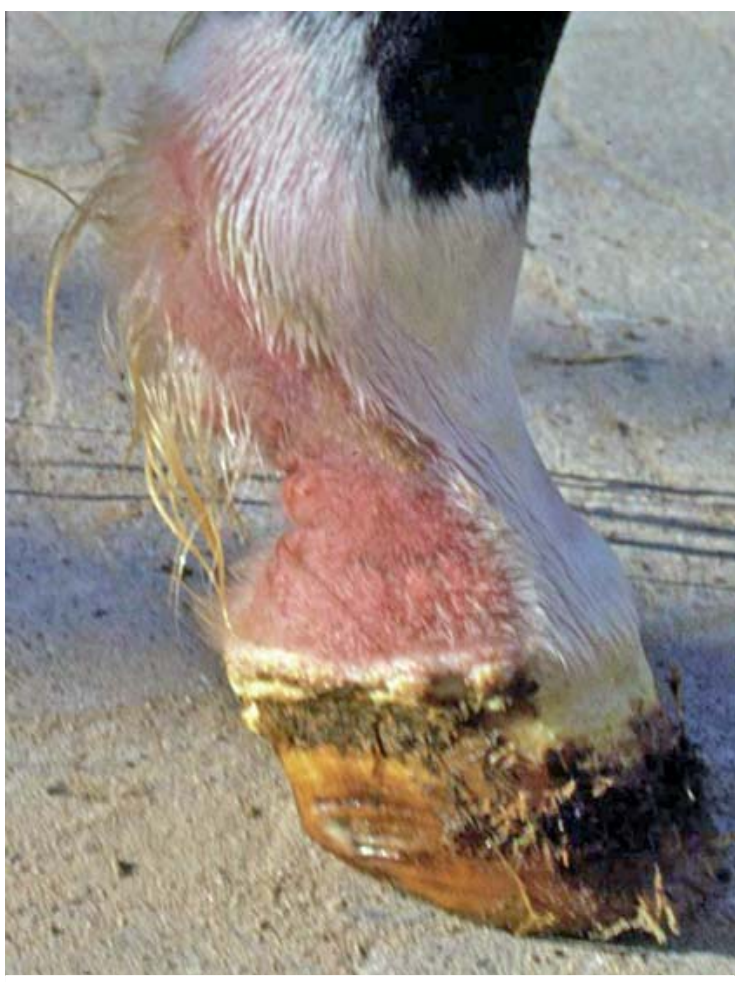

- Abb. 3 Dermatitis im Bereich der Fessel und Fesselbeuge, vermutlich bedingt durch eine leukozytoklastische Vaskulitis. Differenzialdiagnostisch kann diese durch eine medikamentelle Überempfindlichkeitsreaktion ausgelöst werden. Eine weitere Abklärung erfolgte mittels Biopsie. (c) Klinik für Pferde, Stiftung Tierärztliche Hochschule Hannover

rissen der Haut und starken Schuppen beziehungsweise Krustenbildung. Diese Sonderform der Warzenmauke wird auch als „Raspe“ bezeichnet [12].

Erste Anzeichen einer Mauke sind Erytheme, Schuppen, rissige Haut sowie eine unterschiedlich ausgeprägte Berührungsempfindlichkeit. Anschließend kommt es zur Entwicklung von haarlosen Stellen, Erosionen der Haut und Krustenbildung. Bei schwerwiegenden Fällen kann es zur Lymphangitis mit Gliedmaßenschwellung und Fieber kommen. Je nach Ätiologie kann Juckreiz auftreten.

Bei einer chronischen Entzündung kommt es zu hochgradigen Gewebsveränderungen, die irreversibel sind und zu chronischen Lahmheiten führen können.

\author{
ERSTE MAUKEANZEICHEN \\ - Erythem \\ - Schuppen \\ - rissige Haut \\ - Berührungsempfindlichkeit
}



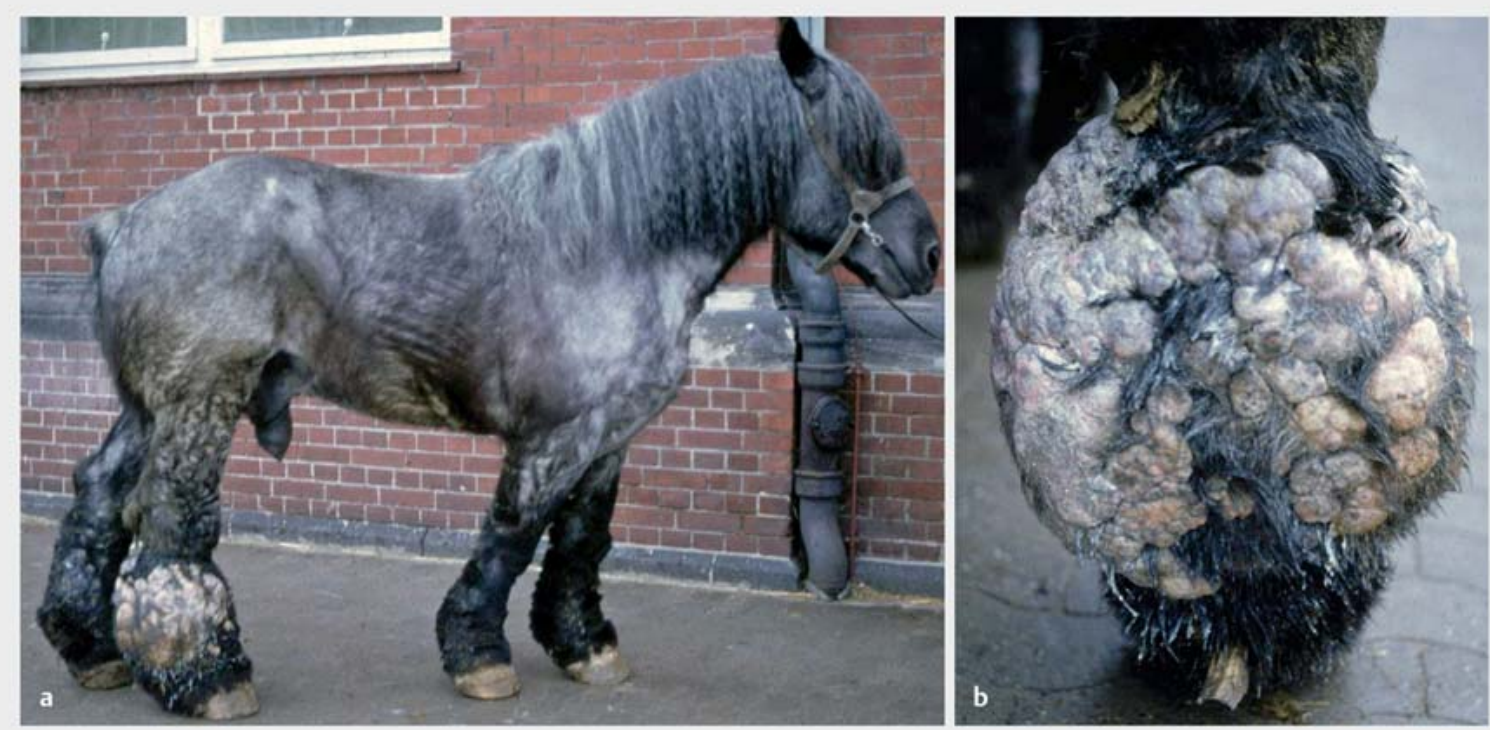

- Abb. 4 a Warzenmauke bei einem Kaltblut. @ Klinik für Pferde, Stiftung Tierärztliche Hochschule Hannover. b Detailaufnahme. ๑) Klinik für Pferde, Stiftung Tierärztliche Hochschule Hannover

Die klinische Präsentation kann grob in 3 Hauptformen unterteilt werden [2]:

1. milde Form (scratches, mud fever) Diese Form tritt am häufigsten auf und ist gekennzeichnet durch Alopezie, trockene Schuppen und Krustenbildung. Die Haut kann gerötet und Juckreiz sowie Schmerzhaftigkeit können eventuell vorhanden sein ( $\bullet$ Abb. 5).

2. exsudative Form (grease heel, dew poisoning) ( $\triangleright$ Abb. 6) Wie der Name schon sagt, ist diese Form deutlich exsudativer mit Erythembildung, Erosionen der Haut sowie Alopezie. Sie ist durch eine serös-purulente, krustöse Dermatitis gekennzeichnet. Histologisch wird sie häufig von Epidermolyse (Blasenbildung) und Vaskulitis begleitet.

3. chronische proliferative Form (grapes, verrucous pododermatitis, Warzenmauke, - Abb.4) Diese chronische Form der Mauke kommt häufig beim Kaltblüter vor. Sie zeichnet sich durch exzessives Granulationsgewebe mit fibroblastischer Proliferation aus, die nodulär Hyperkeratosen und Lichenifikation zeigen kann. Diese stellen traubenartige Wucherungen der Haut dar.

\section{Mögliche Ursachen für Mauke}

Mögliche bakterielle Ursachen für Mauke, die durch eine Follikulitis bzw. Pyodermie gekennzeichnet ist, sind hauptsächlich der Befall mit Staphylococcus aureus und Dermatophilus congolensis. Dabei sind diese eher Sekundärinfektionen und nicht die Primärursache für die Mauke.

\section{Staphylococcus aureus}

Bei einer durch Staphylococcus (Staph.) aureus ausgelösten Dermatitis kommt es zunächst zur Entwicklung von Bläschen, seltener auch Pusteln, die allerdings im späteren Verlauf häufig nicht mehr sichtbar sind.

Nachgewiesen wird die Infektion durch die kulturelle Anzucht. Dafür eignen sich eine steril entnommene Biopsie und die Flüssigkeitsentnahme aus einem Bläschen am besten. Ein Abstrich im späteren Stadium ist oft nicht mehr aussagekräftig, da eine sekundäre bakterielle Besiedlung stattgefunden hat und der ursprüngliche Keim nicht mehr ermittelt werden kann.

\section{Dermatophilus congolensis}

Dermatophilus (D.) congolensis ist eine andere mögliche bakterielle Ursache für Mauke, die teilweise (2/12 Pferden) bei Pferden mit Mauke nachgewiesen werden konnte [10]. Typisch für eine Dermatitis ausgelöst durch D. congolensis sind krustöse und exsudative Hautveränderungen mit ulzerativen Hautarealen unter den Krusten. Die Veränderungen treten typischerweise am Rücken und an der Kruppe auf, können jedoch auch an den Fesseln vorkommen. Voraussetzung für eine klinisch manifeste Infektion ist dabei ein chronisch feuchter Hautzustand mit Reizung der Haut. Die klinische Symptomatik tritt vor allem bei immunsupprimierten und mangelernährten Pferden auf. Typisch für die Infektion sind das pinselartige Verkleben der Haare sowie die ulzerativen Hautveränderungen darunter ( $\bullet$ Abb. 7). 


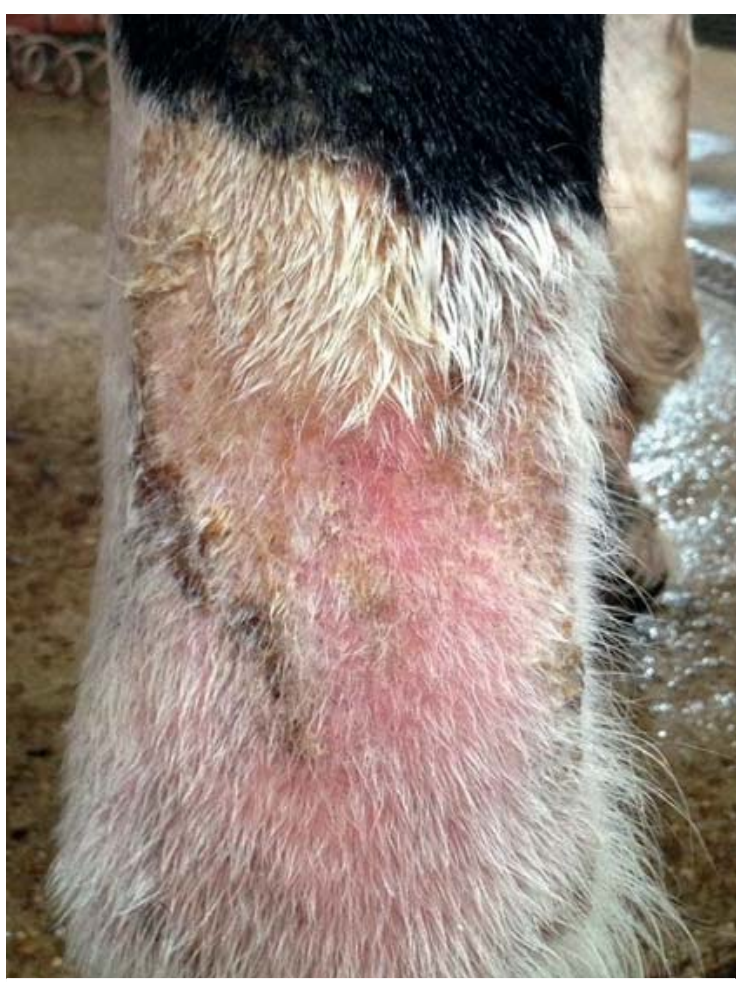

- Abb. 5 Lokale Rötung der Haut im Bereich der Fessel und des unteren Röhrbeins als Frühform der Mauke bei einem Warmblutpferd (Pferd aus $\triangleright \mathbf{A b b} \mathbf{1}$ und $\mathbf{2}$ ).

(c) Charlotte Hopster-Iversen

Der Keim wird mikroskopisch nach Abklatschprobe oder kulturell nachgewiesen.

\section{Dermatophytose}

Eine Dermatophytose (bedingt durch Trichophyton equinum) ist selten die Ursache für Mauke, muss allerdings als wichtige Differenzialdiagnose ausgeschlossen werden.

Der Nachweis gelingt kulturell, nimmt allerdings eine längere Kultivierungszeit in Anspruch. Die Sporen lassen sich teilweise mikroskopisch an den Haarschäften darstellen. Neu ist der Nachweis mittels qPCR (quantitative Echtzeit-PCR), die durch eine hohe Sensitivität und Spezifität gekennzeichnet ist [6]. Ein weiterer Vorteil ist die kurze Nachweiszeit des qPCR (am selben Tag) im Vergleich zur kulturellen Anzucht (2-3 Wochen). Leider ist der Test zurzeit nur am Liphook Equine Hospital in England verfügbar.

\section{Milben}

Ein Befall mit Milben (genauer: Chorioptes spp.) kommt ebenfalls als Ursache für Mauke infrage, vor allem bei Pferden mit Kötenbehang. Dabei zeigen betroffene Pferde starken Juckreiz mit Stampfen und Benagen der Gliedmaßen. Häufig sind mehrere Pferde eines Bestands betroffen.

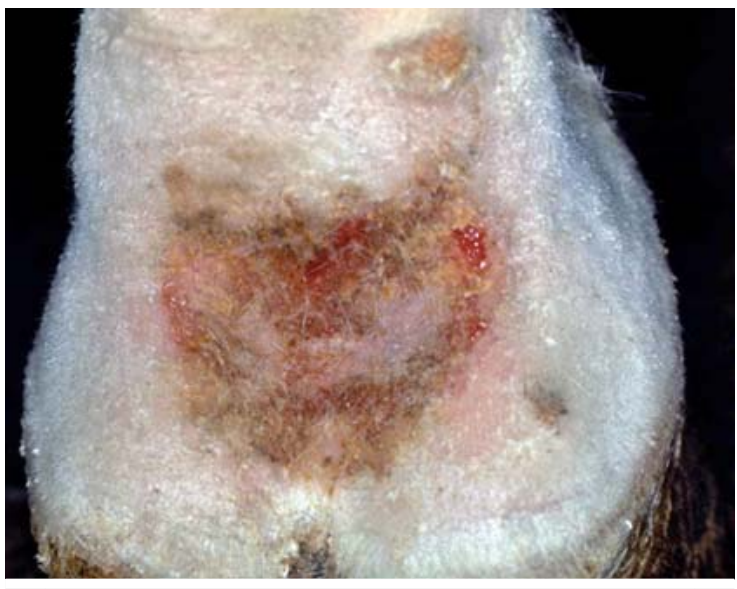

- Abb. 6 Mauke im Bereich der Fesselbeuge mit krustösen und leicht exsudativen Veränderungen. @ Klinik für Pferde, Stiftung Tierärztliche Hochschule Hannover

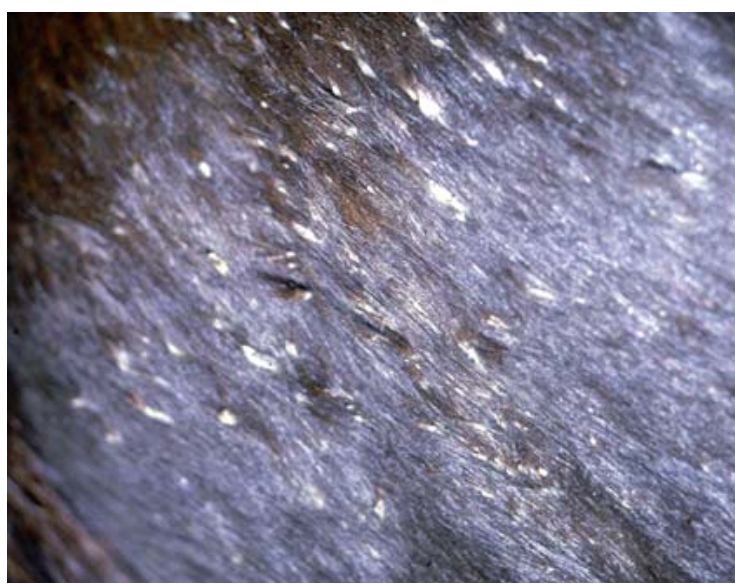

- Abb. 7 Dermatophilose als Differenzialdiagnose zur Mauke. @ Klinik für Pferde, Stiftung Tierärztliche Hochschule Hannover

Der Nachweis gelingt mittels Schabeprobe und mikroskopischer Untersuchung.

\section{Photosensibilisierung}

Eine Photosensibilisierung als Ursache für die Mauke kann systemisch oder durch Kontakt erfolgen: Bei einer primären Photosensibilisierung erreicht ein photodynamischer Stoff entweder direkt die Haut oder gelangt sekundär durch orale Aufnahme bzw. durch Injektion dort hin. Durch den Einfluss von UV-Strahlen und somit der Aktivierung des photodynamischen Stoffs kommt es dann zur Dermatitis. Alternativ kann es bei einer Leberschädigung zur Ansammlung von Phylloerythrinen in der Haut kommen, die ebenfalls nach Aktivierung durch UV-Strahlen zur Dermatitis führen (sekundäre Photosensibilisierung). 
Insgesamt sind bei einer photodynamisch bedingten Dermatitis nur nicht oder wenig pigmentierte Hautareale betroffen, also vor allem weiße Abzeichen (distale Gliedmaßen und Kopf) [13]. Manche Medikamente können auch als Photoallergene agieren und ebenfalls eine Photodermatitis auslösen (z. B. Sulfonamide).

\section{Diagnostische Abklärung}

Bei der diagnostischen Abklärung eines Pferdes mit Mauke ist eine ausführliche Anamneseerhebung wichtig. Dabei werden folgende Punkte abgefragt:

- Art, Typ und Entwicklung der Veränderung

- wann aufgetreten?

- Dauer

- saisonal/nicht saisonal

- Medikation/Produktanwendung vor Auftreten der Veränderungen?

- Kontaktallergie

- Verhalten und Präsentation des Falls

- eine oder mehrere Gliedmaßen

- Juckreiz

- andere Auffälligkeiten

- Schwellung der Gliedmaße

- andere Lokalisationen betroffen (Photodermatitis)

- Haltung des Pferdes

- Box

- Einstreu

- Nässe?

- Weide

- Nässe?

- Paddock

- Sand?

- Vorbehandlung

- lokal

- systemisch

- Effekt?

- evtl. infektiöse/zoonotische Aspekte

- andere Pferde betroffen

- Menschen betroffen (Hautausschlag)

\section{Merke}

Die Anamnese hat in der Maukediagnostik den gleichen Stellenwert wie die klinische Untersuchung.

Nach der Anamneseerhebung kann ggf. bereits eine mögliche Ursache für die Veränderung vermutet werden. Die häufigste Ursache für eine durch Irritation bedingte Dermatitis ist andauernde Feuchtigkeit. Dabei spielen nasse Einstreu oder etwa matschige Weiden und Ausläufe eine wichtige Rolle. Auch führt der Behang bei Kaltblutpferden zu einer permanenten Feuchtigkeit auf der Haut und kann zu einer chronischen Aufweichung der Haut führen.
Bei einer Kontakt- oder Irritationsdermatitis sind meistens alle 4 Gliedmaßen betroffen. Wenn andere Pferde oder Menschen ebenfalls Hautveränderungen aufweisen, muss von einer möglichen infektiösen Ursache (wie z.B. Dermatophyten) ausgegangen werden.

Nach der ausführlichen Anamneseerhebung erfolgt die Untersuchung des Patienten:

- klinische Untersuchung

- spezielle klinische Untersuchung der Haut

- Identifizierung von primären Läsionen

- Identifizierung von sekundären Läsionen

Hieran schließen sich ggf. weiterführende Untersuchungen an:

\section{Diagnostische Tests}

Abschabepräparat

Mithilfe einer Skalpellklinge oder eines scharfen Löffels werden Hautschuppen sowie Krusten abgeschabt und aufgefangen. Anschließend werden die Schuppen mittels Öl fixiert und unterm Mikroskop untersucht (10-fache Vergrößerung). Diese Methode dient vor allem dem Nachweis oder Ausschluss von Milben (Chorioptes spp).

\section{Tesafilmabklatschpräparat mit Diff-Quick-Färbung}

Betroffene Hautareale werden mittels Tesafilm beprobt. Das Präparat kann direkt unter dem Mikroskop (Milben) oder nach vorheriger Diff-Quick-Färbung untersucht werden. Dann können ggf. Bakterien (Kokken) sowie degenerative neutrophile Granulozyten (Pyodermie) oder Hefen (Malassezia) nachgewiesen werden [2]. Sollten neutrophile Granulozyten (nicht vakuolisiert) mit fehlenden oder nur wenigen Bakterien vorhanden sein, kann das ein Hinweis auf eine auto-immunbedingte Ursache sein. Diese sollte mittels Biopsie näher untersucht werden. Ebenso bei einer homogenen Zellpopulation mit erhöhter Teilungsrate und atypischem Zellkern-Zytoplasma-Verhältnis, die ein Hinweis auf eine neoplastische Ursache sein können. Eosinophile Granulozyten können auf eine allergische, parasitäre oder mykotische Ursache oder einen Fremdkörper hindeuten.

\section{Haaranalyse zum Nachweis von Dermatophyten}

Haare betroffener Areale werden mit ihren Wurzeln entnommen und unter dem Mikroskop nach Zugabe von 12 Tropfen 10\% Kaliumhydroxid (KOH) zur Aufhellung mit einem Deckglas untersucht. Betroffene Haare sind blass und geschwollen und haben Arthrosporen im Bereich des Haarschafts. Der Nachweis ist zeitaufwändig und erfordert einige Routine.

\section{kultureller Keimnachweis}

Der kulturelle Nachweis von Bakterien (Staph. aureus, D. congolensis) und Hefen erfolgt auf Spezialkulturen. Hierfür ist eine Biopsie am besten geeignet. Die Entnahme erfolgt nach chirurgischer Vorbereitung des zu be- 
probenden Areals (Scheren und Waschung mit Chlorhexidin oder Jodseife).

\section{Biopsie zur histopathologischen Untersuchung}

Eine histopathologische Untersuchung ist sinnvoll, wenn eine immun-mediierte oder neoplastische Ursache für die Mauke vermutet wird. Dabei sollten möglichst Primärläsionen beprobt werden, um akute Veränderungen wie eine leukozytoklastische Vaskulitis, Thrombosen oder Gefäßwandnekrosen zu identifizieren.

\section{Blutuntersuchung}

Zum Ausschluss einer Lebererkrankung und daraus resultierender Photosensibilisierung ist die Messung der leberspezifischen Enzymaktivitäten von GLDH, GGT und der Gallensäurenkonzentration sinnvoll.

\section{Gentest}

Beim Kaltblut wurde mittels Genotypisierung ein Teil eines Chromosoms identifiziert, das Einfluss auf die Ausprägung von Warzenmauke hat (quantitative trait locus) [7]. Dies ermöglicht zu einem späteren Zeitpunkt eventuell einen Screening-Test für Warzenmauke.

\section{Therapieoptionen}

Die Auswahl einer passenden Therapie beinhaltet die Identifizierung von prädisponierenden, primären sowie begünstigenden Faktoren und eine mögliche Elimination bzw. Reduktion. Insgesamt kann die Therapie je nach Ausmaß und Schweregrad der Veränderungen langwierig sein. Im Folgenden werden allgemeine Überlegungen zum Management des Pferdes sowie spezielle medikamentelle Therapieoptionen besprochen.

\section{Merke}

Die Therapie der Mauke ist abhängig von ihrer Ursache, schließt aber auch immer eine Optimierung des Managements mit ein.

\section{Umgebung}

- Weiden und Ausläufe mit Matsch, Wasser oder Sand können Mauke hervorrufen bzw. verschlimmern

- Aufstallen der Pferde in einer trockenen Umgebung während nasser Witterung

- Pferde erst nach Abtrocknen des Morgentaus auf die Weide stellen

- bei Verdacht auf eine Kontaktallergie durch die Einstreu über alternative (unbehandelte) Einstreu nachdenken

- starken Behang scheren

- bei Verdacht auf Photosensibiliserung UV-Licht durch Aufstallen oder Einbandagieren empfindlicher Bereiche meiden

- betroffene Areale sollten direkt nach der Bewegung mittels Chlorhexidin-Shampoo gereinigt werden, um den Schweiß zu entfernen

\section{Medikamentelle Therapie}

Eine Übersicht der medikamentellen Therapie findet sich in $>$ Tab. 2 und $\mathbf{3}$.

\section{Topische antibakterielle Therapie}

Bei einer Mauke kommt es häufig zu sekundären bakteriellen Infektionen vor allem mit Staph. aureus. Daher ist eine lokale Therapie mittels Waschungen empfehlenswert. Bei Anwendung eines Shampoos sollte dies ca. 10 Minuten einwirken und der Bereich nach dem Ausspülen gut getrocknet werden. Zunächst ist eine tägliche Anwendung zu empfehlen. Nach ca. 1 Woche kann die Anwendung auf 2-3 Mal wöchentlich reduziert werden.

Als Wirkstoffe kommen infrage:

- Chlorhexidin $(2 \%)^{1}$

- Benzoylperoxid ${ }^{2}$

- Ethyllactat ${ }^{1}$

Weiterhin können Lotionen mit antimikrobieller Wirkung angewendet werden:

- Silbersulfadiazin ${ }^{2}$

- $1 \%$ Silbersulfadiazin ${ }^{2}$ kombiniert mit 0,2\% Chlorhexidindiglukonat

- Mikrosilber ${ }^{1}$

- Kanuka-Öl [8]

- als Mischung mit Salicylöl ${ }^{1}$ wirksam gegen Staph. aureus, andere gram-positive Bakterien sowie gegen Dermatophyten

- bei 7/10 Pferden vollständige Heilung nach 7 Tagen Behandlungsdauer

- Präparat in Deutschland zum aktuellen Zeitpunkt nicht erhältlich und eigene Herstellung nicht erlaubt

- $1 \%$ Wasserstoffperoxidcreme ${ }^{1}$

- Honig

- helles sulfoniertes Schieferöl (Natriumbituminosulfonat oder Ichtyol) ${ }^{1}$

- wundheilungsfördernd

- juckreizlindernd

- austrocknend

- Mupirocin ${ }^{2}$ Bactoderm $^{\circledR}$, Pfizer

- antibakteriell und hautpenetrierend

- Fusidinsäure ${ }^{2}$

- bakteriostatisch

- Zinnfluorid (0,4\%) $1 \times$ täglich MedEquine ${ }^{\circledR}$ [9]

- bakterizid

- Oxychlorid²-Spray (Veterycin ${ }^{\mathrm{TM}}$ ) (mehrfach täglich)

- bakterizid

Die einzelnen Wirkstoffe sind je nach aktueller Gesetzeslage entsprechend ihrer Anwendungszulassung gekennzeichnet für bei: ${ }^{1}$ Lebensmittel-liefernden Tieren zugelassen; ${ }^{2}$ nicht bei Lebensmittel-liefernden Tieren zugelassen, ${ }^{2 b}$ in der Positivliste; ${ }^{3}$ in der Verordnung über apothekenpflichtige und freiverkäufliche Arzneimittel 
- Tab. 2 Wirkstoffe und ihre Wirkungsweisen zur Behandlung der Mauke bei Pferden.

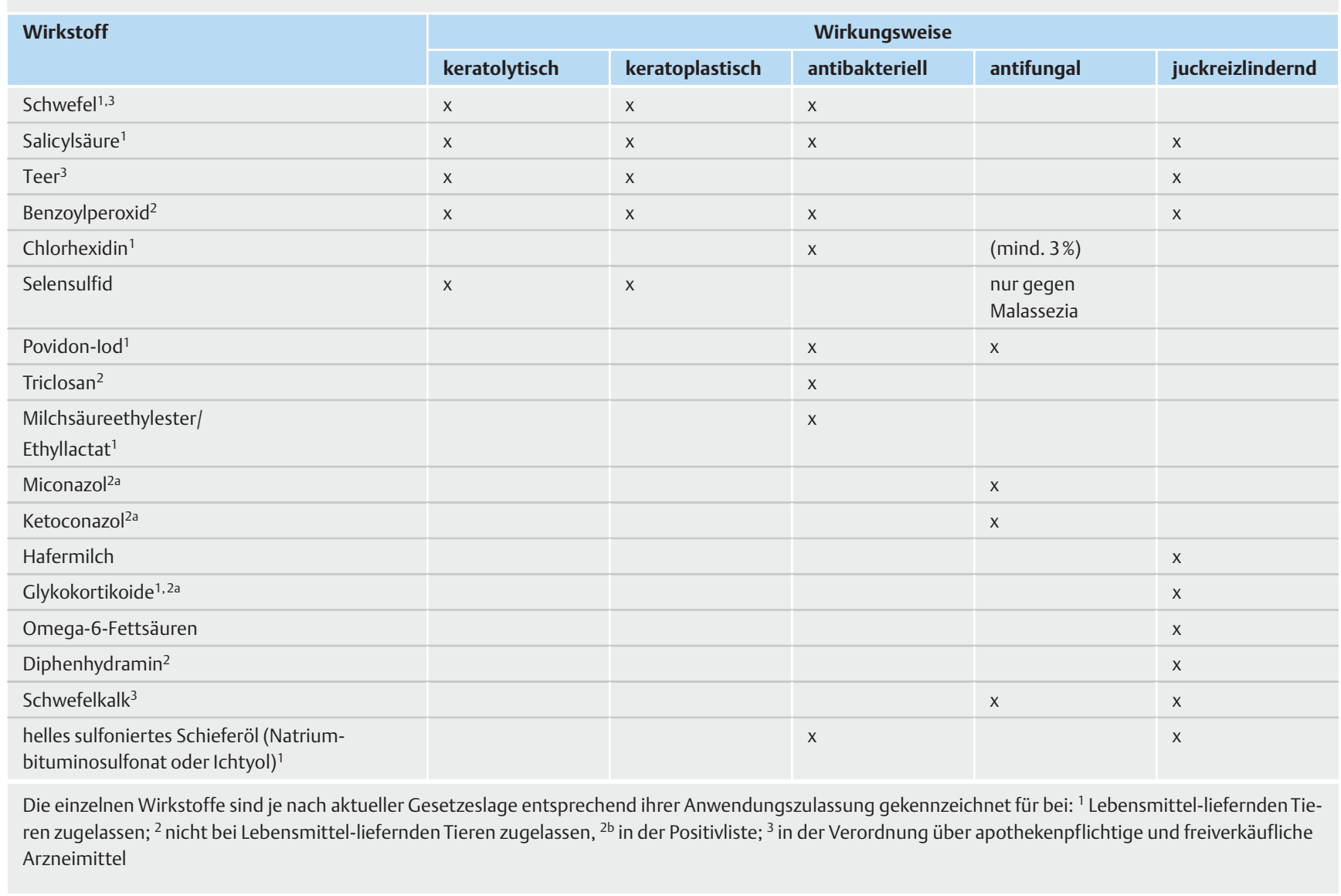

- Equitech ${ }^{\circledR}$ Mud Stop [10]

- Kombinationspräparat aus wasserabweisenden (Monopropylenglykol und Isopropanol) und antibakteriell wirksamen (Triclosan) Agenzien; wirksam bei 10/12 Pferden mit Mauke und nachgewiesener bakterieller Besiedlung.

Zusätzlich zur lokalen medikamentellen Therapie ist Hautschutz von großer Bedeutung. Betroffene Areale sollten eventuell mittels einer wasserabweisenden Bandage vor Nässe geschützt werden.

\section{- adstringierende Substanzen:}

Bei exsudativen Veränderungen kann die Anwendung von adstringierenden Lösungen hilfreich sein. Dabei sind folgende Wirkstoffe empfehlenswert:

- Aluminiumacetat ${ }^{1}$-Lösung

- Schwefelkalk ${ }^{3}$

- je nach Grad der Krustenbildung kann eine Aufweichung der borkigen Haut sinnvoll sein. Dabei unterscheidet man folgende Eigenschaften bei den Wirkstoffen:

- keratolytisch: löst die äußere Schicht der Haut durch Beeinflussung der Korneozyten
- keratoplastisch: normalisiert die Keratinisierung durch Verringerung der Teilungsrate der Basalzellmembran

In manchen Fällen ist eine Verbandstherapie, zumindest für die Anfangstherapie, indiziert. Dies gilt insbesondere, wenn eine ödematöse, ggf. infizierte Schwellung der Gliedmaße vorliegt oder wenn besonders hartnäckige Krusten gelöst werden sollen. Eine Gefahr von Mauke ist je nach Ausmaß der Veränderung immer eine Infektion der Gliedmaße bis hin zur Phlegmone oder chronische ödematöse Schwellung. Dem kann mittels einer lokalen Verbandstherapie entgegengewirkt werden. Dabei werden häufig zunächst Angussverbände angelegt, z. B. mit verdünnter lodlösung ${ }^{1}$ oder Acridinlösung ${ }^{2}$, gefolgt von trockenen Salbenverbänden.

In seltenen schwerwiegenden Fällen ist eine zusätzliche systemische antimikrobielle Therapie sinnvoll:

- Procain-Penicillin ${ }^{1}$

- Breitband-Antibiotika oder bei kulturellem Nachweis von gram-negativen Keimen selektiv gegen gram-negative Erreger 
\ Tab. 3 Ergänzung zu den Kennziffern bezüglich der Arzneimittel aus $\$ Tab. 2.

\begin{tabular}{|c|c|c|c|}
\hline $\begin{array}{l}\text { Kennziffer } 1 \\
\text { Genannt in Tabelle } 1 \text { der Vo } \\
\text { (EU) Nr. 37/2010, damit zur } \\
\text { Anwendung bei Lebensmittel } \\
\text { liefernden Tierarten erlaubt }\end{array}$ & $\begin{array}{l}\text { Kennziffer } 2 \\
\text { Nicht in der VO (EU) Nr. 37| } \\
2010 \text { aufgeführt und damit } \\
\text { nicht bei Lebensmittel liefern- } \\
\text { den Tieren erlaubt }\end{array}$ & $\begin{array}{l}\text { Kennziffer 2b } \\
\text { Genannt in der VO (EG) Nr. 122| } \\
2013 \text { (vorher VO (EG) Nr. 1950| } \\
2006) \text { : Verzeichnis, der zur Be- } \\
\text { handlung von Equiden wesentli- } \\
\text { chen Stoffe (sog. Positivliste für } \\
\text { Equiden). } \\
\text { Die Anwendung ist gemäß wei- } \\
\text { terer europäischer Rechtsvor- } \\
\text { schriften durch eine Eintragung im } \\
\text { Equidenpass zu dokumentieren } \\
\text { und eine Wartezeit von mindes- } \\
\text { tens } 6 \text { Monaten ist einzuhalten. }\end{array}$ & $\begin{array}{l}\text { Kennziffer } 3 \\
\text { Wirkstoffe, die in der Ver- } \\
\text { ordnung über apotheken- } \\
\text { pflichtige und freiverkäuf- } \\
\text { liche Arzneimittel (BGBI. I } \\
\text { S. 2371) genannt sind }\end{array}$ \\
\hline $\begin{array}{l}\text { - Aluminiumacetat } \\
\text { - Chlorhexidin } \\
\text { - Ethyllactat } \\
\text { - gewisse Glukokortikoide } \\
\text { - Ichthyol } \\
\text { - lod } \\
\text { - Procain-Penicillin } \\
\text { - Salicylsäure } \\
\text { - Silber (E 174) } \\
\text { - Schwefel } \\
\text { - Wasserstoffperoxid }\end{array}$ & $\begin{array}{l}\text { - Benzoylperoxid } \\
\text { - Im Therapienotstand kann } \\
\text { ein Tierarzneimittel um- } \\
\text { gewidmet werden, z. B. } \\
\text { Peroxyderm (Vetoquinol), } \\
\text { zugelassen für Hunde } \\
\text { - Diphenhydramin } \\
\text { - Fusidinsäure } \\
\text { - Im Therapienotstand kann } \\
\text { ein Tierarzneimittel um- } \\
\text { gewidmet werden, z. B. } \\
\text { Isaderm (Albrecht), zuge- } \\
\text { lassen für Hunde } \\
\text { - Mupirocin } \\
\text { - Als Tierarzneimittel Bacto- } \\
\text { derm verfügbar, allerdings } \\
\text { nicht in Deutschland } \\
\text { - Oxychlorid } \\
\text { - Silbersulfadiaxin } \\
\text { - Im Therapienotstand kann } \\
\text { ein Arzneimittel aus der Hu- } \\
\text { manmedizin umgewidmet } \\
\text { werden }\end{array}$ & $\begin{array}{l}\text { - Miconazol (Miconazol kann bei } \\
\text { Schlachtequiden (nicht aber bei } \\
\text { anderen Lebensmittel liefernden } \\
\text { Tierarten!) eingesetzt werden.) } \\
\text { - Ketoconazol } \\
\text { - gewisse Glukokortikoide }\end{array}$ & $\begin{array}{l}\text { - Holzteer } \\
\text { - Schwefel }\end{array}$ \\
\hline
\end{tabular}

Bei Verdacht auf auto-immunbedingte Veränderungen (leukozytoklastische Vaskulitis) ist eine Initialtherapie mit Glukokortikoiden notwendig. Dabei ist Dexamethason ${ }^{1}$ initial indiziert $(0,1 \mathrm{mg} / \mathrm{kg}$ i.v oder i. m. $1 \times$ tgl.), gefolgt von einer ausschleichenden Therapie mit Prednisolon ${ }^{1}$ oral $(1(-2) \mathrm{mg} / \mathrm{kg}$ oral $1 \times$ tgl. $)$.

Bei der chronischen Warzenmauke, bei der der gestörte Lymphabfluss eine wichtige Rolle spielt, können unterstützend manuelle Lymphdrainage sowie anschließend Kompressionsstrümpfe sinnvoll sein. Dies führte in einer Studie der University of California Davis zur Verminderung des Gliedmaßenumfangs sowie zur Verbesserung der Mobilität [11].

\section{Hinweis zur Arzneimittelverordnung}

Sowohl bei der lokalen wie auch systemischen medikamentellen Therapie muss die entsprechende Arzneimittelverordnung beachtet werden. Die einzelnen Wirkstoffe der $>$ Tab. 2 sind nach der aktuellen Gesetzeslage entsprechend ihrer Anwendungszulassung mit beim Lebensmittelliefernden Tier zugelassen $\left({ }^{1}\right)$, nicht beim LMliefernden Tier zugelassen $\left({ }^{2}\right)$, Positivliste $\left({ }^{2 b}\right)$ oder Verordnung über apothekenpflichtige und freiverkäufliche Arzneimittel ( ${ }^{3}$ ) soweit auffindbar gekennzeichnet.

\section{Prognose}

Je nach Schweregrad der Mauke ist die Prognose als gut bis vorsichtig anzugeben. Bei Pferden mit Warzenmauke ist eine vollständige Heilung oft unwahrscheinlich, allerdings können die Symptome durch konsequente Pflege und Behandlung bei ersten Auffälligkeiten abgemildert werden. Bei Pferden mit einer milden Form der Mauke ist die konsequente Kombination aus Haltungsoptimierung zusammen mit einer gezielten und konsequenten lokalen Behandlung oft erfolgreich, jedoch ist die Therapie häufig langwierig und die Rezidivgefahr hoch. 
Dr. Charlotte Hopster-Iversen, PhD, Dipl. ECEIM

Penn Vet Preclinical Service Core \&

Comparative Orthopedic Research Laboratory

University of Pennsylvania

School of Veterinary Medicine

Department of Clinical Studies-New Bolton Center

382 West Street Road

Kennett Square, PA 19348

USA

\section{Literatur}

[1] Scott DW, Miller WH Jr. Pastern dermatitis. In: Scott DW, Miller WH, eds. Equine Dermatology, 2nded. Maryland Heights: Saunders Elsevier 2011; 260-261

[2] Yu AA. Equine pastern dermatitis. Vet Clin North Am Equine Pract 2013; 29: 577-588

[3] Federici M, Gerber V, Doherr MG et al. Association of skin problems with coat colour and white markings in three-yearold horses of the Franches-Montagnes breed. Schweiz Arch Tierheilkd 2015; 157: 391-398

[4] Wallraf A, Hamann H, Deegen E et al. Analysis of the prevalence of pastern dermatitis in German coldblood horse breeds. Berl Munch Tierarztl Wochenschr 2004; 117:148-152

[5] Geburek F, Ohnesorge B, Deegen E et al. Alterations of epidermal proliferation and cytokeratin expression in skin biopsies from heavy draught horses with chronic pastern dermatitis. Vet Dermatol 2005; 16(6): 373-384

[6] Durham A. Assessment of the diagnostic value of a novel Dermatophyte QPCR assay in horses. Proceedings, 10th ECEIM Congress 2017, Budapest, Ungarn 2017: 3-12

[7] Mittmann EH, Mömke S, Distl O. Whole-genome scan identifies quantitative trait loci for chronic pastern dermatitis in German draft horses. Mamm Genome 2010: 21(1-2): 95-103

[8] Thomas J, Narkowicz C, Peterson GM et al. Randomised controlled trial of the treatment of pastern dermatitis with a formulation containing kunzea oil. Vet Rec 2009; 164(20): 619-623

[9] Marsella, R. and Akucewich, L. (2007). Investigation on the clinical efficacy and tolerability of a $0.4 \%$ topical stannous fluoride preparation (MedEquine ${ }^{\circledR}$ Gel) for the treatment of bacterial skin infections in horses: a prospective, randomized, double-blinded, placebo-controlled clinical trial. Veterinary dermatology, 18(6), 444-450

[10] Colles CM, Colles KM, Galpin JR. Equine pastern dermatitis. Equine Vet Educ. 2010; 22: 566-570

[11] Powell H, Affolter VK. Combined decongestive therapy including equine manual lymph drainage to assist management of chronic progressive lymphoedema in draught horses. Equine Vet Educ 2012; 24(2): 81-89

[12] Geburek, F. Zur Entstehung des Warzenmauke-Syndroms der Kaltblutpferde-klinische und histomorphologische Untersuchungen. Diss. med. vet., Tierärztliche Hochschule Hannover, 2002

[13] Dahme E, Weiss E. Grundriss der speziellen pathologischen Anatomie der Haustiere. Stuttgart: Enke 2007

Bibliografie

DOI https://doi.org/10.1055/s-0044-100330

Pferdespiegel 2018; 21: 136-147

(c) Georg Thieme Verlag KG Stuttgart · New York

ISSN 1860-3203 
CVE-Fragebogen

\section{Frage 1}

Welches der folgenden Bakterien ist häufig mit ursächlich für Mauke als Sekundärinfektion?
a Staph. aureus
b Strept. equi spp. equi
c Strept. suis
d Pseudomonas aeruginosa
e E. coli

\section{Frage 2}

Welche der folgenden Pferderassen ist häufiger von Mauke betroffen?
a Isländer
b Shetlandpony
c Schleswiger Kaltblut
d Arabisches Vollblut
e Oldenburger

\section{Frage 3}

Welcher der folgenden Faktoren erhöht nicht die Prävalenz für Mauke?
a zunehmendes Alter
b Behang
c weiße Gliedmaßen
d Kaltblut
e Geschlecht

\section{Frage 4}

Welche Milbenart kann bei Mauke mitbeteiligt sein und ist am ehesten in der Fessel vorzufinden?
a Sarkoptes
b Chorioptes
c Dermanyssus gallinae
d Demodex canis
e Psoroptes

\section{Frage 5}

Welche der folgende Nachweismethoden eignet sich zum Nachweis von Milben, die den Fesselbereich besiedeln können?
a Abschabepräparat
b Kotuntersuchung
c Abklatschpräparat und mikrobiologische Untersuchung
d Haaranalyse
e Woodsche Lampe

\section{Frage 6}

rtbildun

\section{ATF}

Welcher der folgenden Wirkstoffe hat eine antibakterielle Wirkung?
a Dexpanthenol
b Urea
c Natriumbituminosulfonat
d Prednisolon
e Diphenhydramin

\section{Frage 7}

Welche von den folgenden Wirkstoffen hat eine juckreizlindernde Wirkung?
a Hafermehl
b Chlorhexidin
c lod
d Ketoconazol
e Miconazol

\section{Frage 8}

Sie haben den Verdacht einer leukozytoklastischen Vaskulitis als Ursache für die maukeähnlichen Veränderungen. Wie können Sie die Diagnose absichern?

a durch die Anamneseerhebung

b durch eine diagnostische Ausschlusstherapie mit Chlorhexidinwaschungen

c durch ein Abschabepräparat

d durch eine Biopsieentnahme

e durch eine Blutuntersuchung

\section{Frage 9}

Sie haben die Verdachtsdiagnose einer leukozytoklastischen Vaskulitis bestätigt. Welche Initialtherapie ist jetzt indiziert?
a Dexamethason
b Natriumbituminosulfonat
c Dexpanthenol
d Honig
e Kanuka-Öl

\section{Frage 10}

Welche der folgenden zusätzlichen Therapien kann bei der Warzenmauke sinnvoll sein?
a manuelle Lymphdrainage
b Massage
c Physiotherapie
d Inhalation
e Infrarotbestrahlung 


\section{Mauke beim Pferd}

Charlotte Hopster-Iversen

\section{ATF}

\section{A Lernerfolgskontrolle}

\section{Bitte kreuzen Sie die richtigen \\ Antworten an! Es ist jeweils nur 1 Antwort pro Frage richtig!}

\begin{tabular}{ll|l|l|l|l|l|} 
Frage 1 & a & b & c & d & e \\
Frage 2 & a & b & c & d & e \\
\hline Frage 3 & a & b & c & d & e \\
\hline Frage 4 & a & b & c & d & e \\
\hline Frage 5 & a & b & c & d & e \\
\hline
\end{tabular}

\begin{abstract}
Frage 6 a

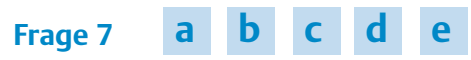

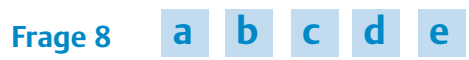

Frage 9 a b c d e

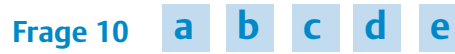

\section{B Teilnehmer}

Titel | Name | Vorname
Straße | Hausnummer

Beruf
PLZ | Ort $\square$ bestanden und 1 ATF-Stunde erhalten

Stuttgart, den $\square$ nicht bestanden.

Stempel | Unterschrift

\section{Teilnahmebedingungen für Abonnenten des pferde.spiegel kostenlos}

Für diese Fortbildung können Sie 1 ATF-Fortbildungsstunde anerkannt bekommen. Hierfür - müssen mindestens $70 \%$ der Fragen richtig beantwortet sein.

- muss der Antwortbogen vollständig ausgefüllt sein. Unvollständig ausgefüllte Bögen können nicht berücksichtigt werden.

\begin{abstract}
- muss im markierten Feld* Ihre Abonnentennummer eingetragen oder eine Pferdespiegel-CVE-Wertmarke aufgeklebt sein.
\end{abstract}

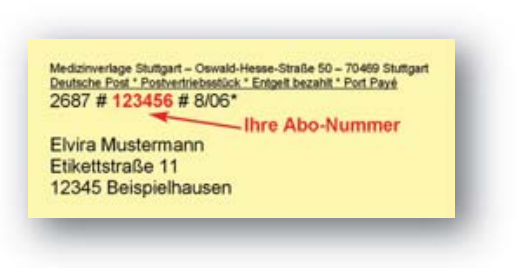

\section{E Erklärung}

Ich versichere, dass ich die Beantwortung der Fragen selbst und ohne fremde Hilfe durchgeführt habe.

Unterschrift
Pferdespiegel-CVE-Wertmarken für Nichtabonnenten können beim Verlag zu folgenden Bedingungen erworben werden: 4er-Pack Wertmarken, Preis 49,95€ inkl. MwSt., ArtikelNr. 903001. Bitte richten Sie die Bestellungen an: Georg Thieme Verlag KG, KundenServiceCenter Buch, Postfach 301120, 70451 Stuttgart.

* Nichtabonnenten bitte hier Pferdespiegel-CVE-Wertmarke aufkleben. Abonnenten bitte Abonnentennummer eintragen: 